\section{Cliff Shaw Award}

The 1991 Cliff Shaw Award for an article of special distinction in the past four issues of Blue Jay was awarded to Jean Bancroft of Winnipeg, Manitoba. Her article, "The range expansion of the House Finch into the Prairie Provinces," involved a tremendous amount of searching published material and obtaining unpublished records of this bird from the Pacific and Atlantic coasts and into the interior. It is an excellent example, along with her many other articles in Blue Jay, of what a serious amateur birder can do. Congratulations are also due to Robert Parsons, who helped Jean with the article.

\section{Larry Morgotch and Conservation Awards}

Don Hooper of Somme took home two awards, the 1991 Conservation Award and the 1991 Larry Morgotch Award. Don received the Conservation Award for his initiative in the acquisition of the Rendek Elm Forest east of Hudson Bay. Don spearheaded the work in acquiring the land and played a major role in setting up trails and facilities at the forest. One of the founding members of the Hudson Bay Natural History Society and a long-time member of the provincial society, Don is an "all round conservationist."

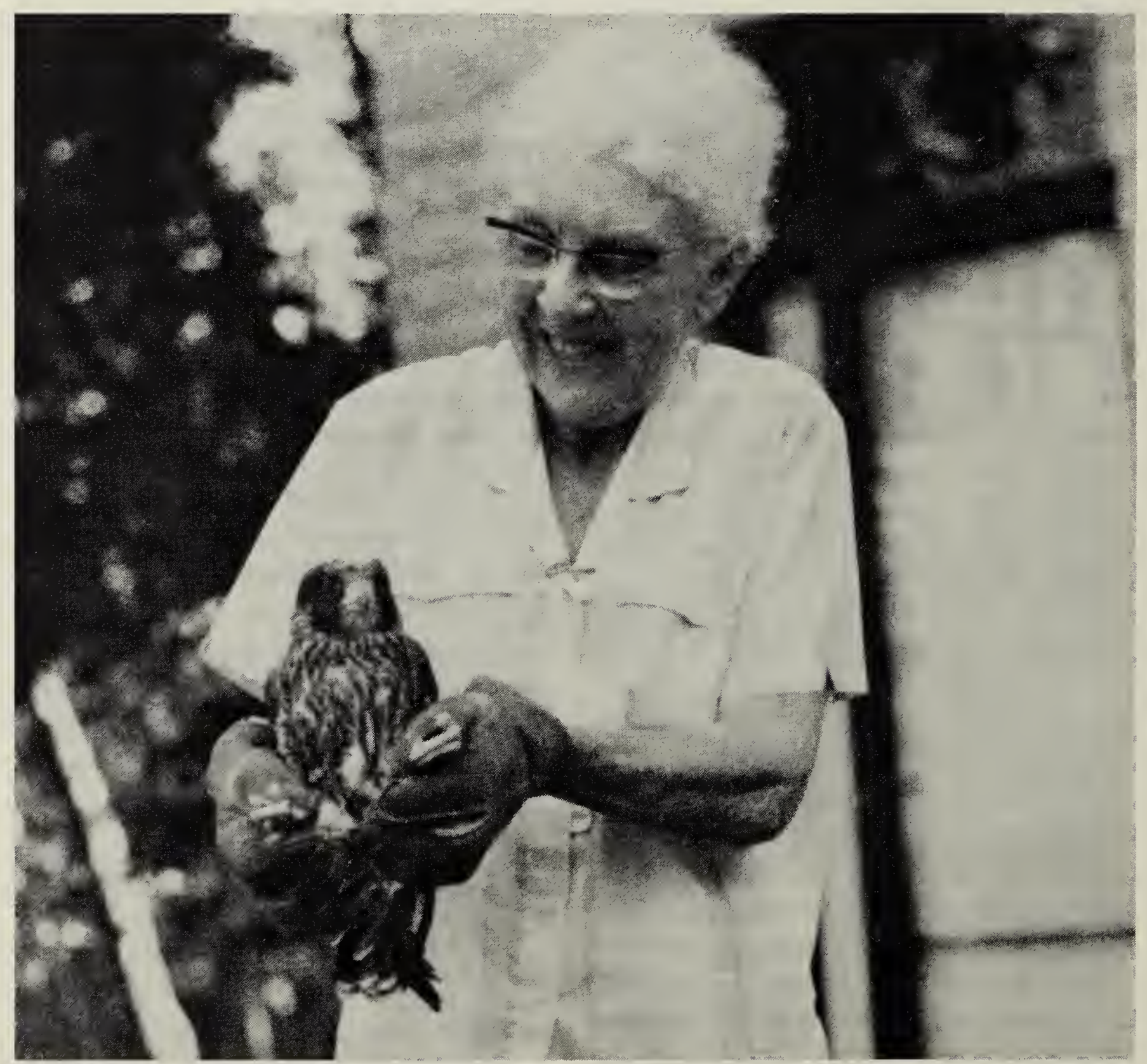

Jean Bancroft, winner of the Cliff Shaw Award, pictured here with a 35-day-old Peregrine Falcon chick. 


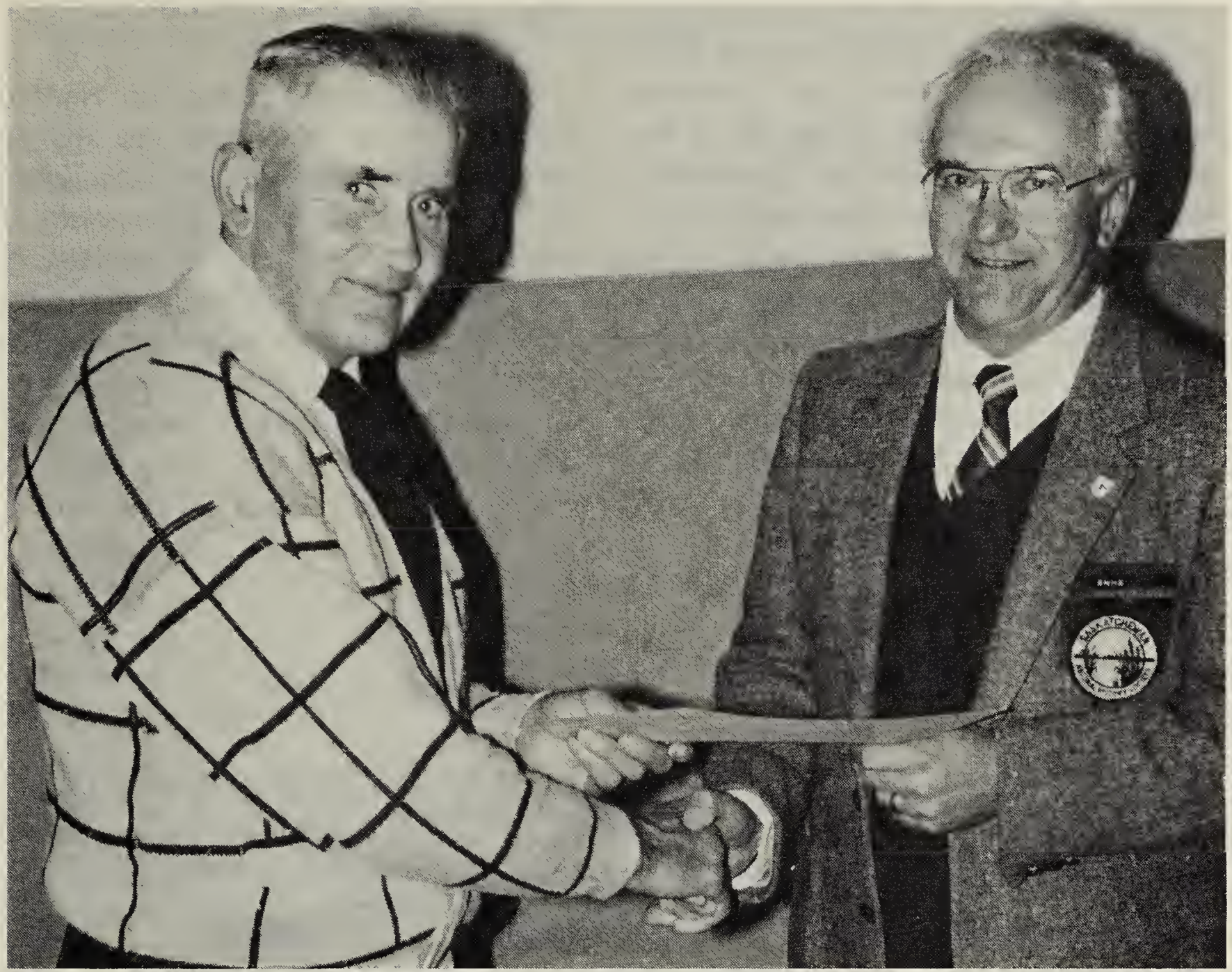

Frank Switzer presents the Larry Morgotch Award to Don Hooper.

Curt Schroeder

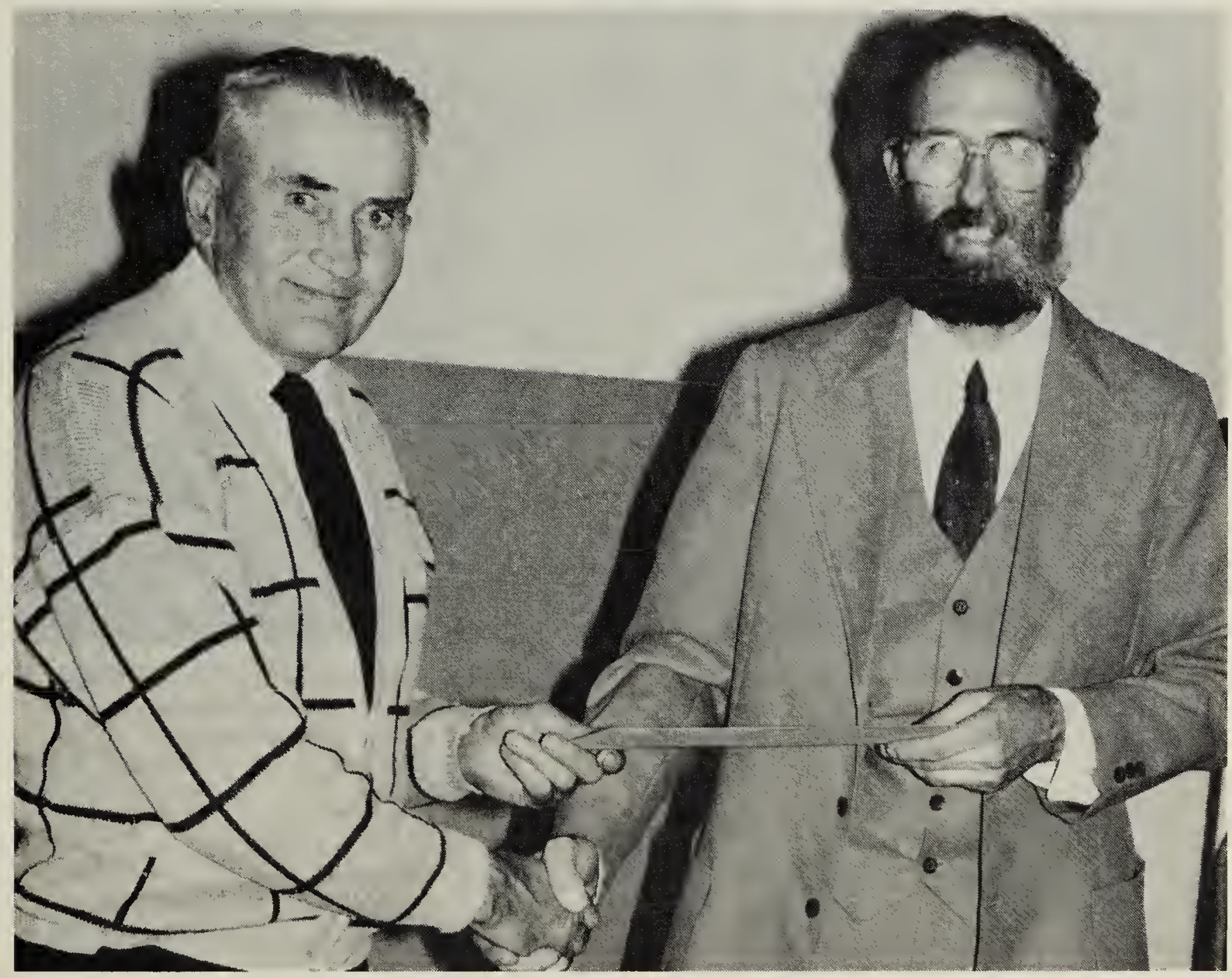


Don is also an avid amateur photogapher. The theme of his excellent slide presentation was how the Goldencrowned Kinglet takes advantage of the work of a Yellow-bellied Saspsucker when the weather is uncooperative by feeding on sap from holes made by the sapsucker (see page 201 - Sap for Survival). The presentation won Don the 1991 Larry Morgatch Award for the best photographic story and the best photograph entered in the annual competition.

\section{Weyburn Birdathon}

On behalf of the Saskatoon Natural History Society, President Pat Adams accepted the award for the most birds (277 species) on a list for a club or society in the Weyburn Natural History Society's Provincial Summer Count 1991. Burke Korol of Saskatoon won the award for the most species identified by an individual. Burke counted 247 different species.

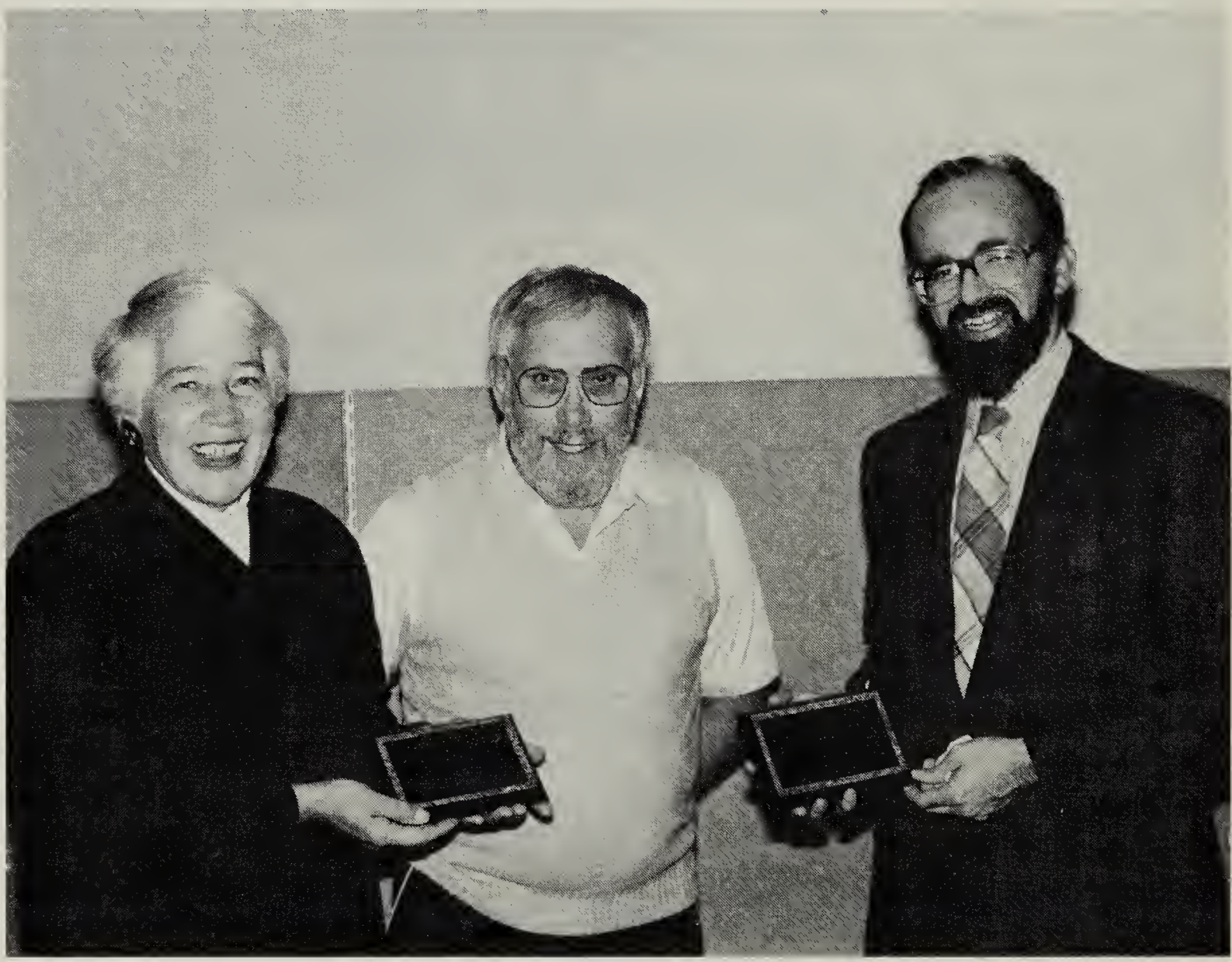

Pat Adams accepts the award for Most Birds on a Club List for Saskatoon N.H.S., from Dick Gutfriend of the Weyburn N.H.S. Stan Shadick accepted the award for Most Birds on an Individual's List in the Weyburn Birdathon. Stan accepted on behalf of Burke Korol.

Frank Switzer 
REVENUE

1991

1990

Grants - Sask Trust for Sport, Culture

and Recreation: Annual Global Funding

$\$ 100,000$

$\$ 100,000$

Grants - Specified Programs

151,440

27,175

Grants - Other

Specified Programs

5,364

15,000

Bequest Trust

2,000

Self Generated

Advertising

450

580

Conservation Sales

1,498

1,073

Donations

3,636

Interest

3,696

12,938

Memberships

10,765

19,334

Special Publications

18,197

774

1,038

Spring Meet

1,584

Tour Income

5,056

1,140

301,964

6,801

Recovery - Loggerhead Shrike

$19 \overline{0,125}$

\section{EXPENSES}

Administrative Salaries and Benefits

Office - Schedule 2

28,553

27,874

Membership Communications and Publications

Blue Jay Journal

18,223

19,049

5,955

7,189

Travel and Meetings

Staff and Volunteer Travel

2,044

5,761

1,613

421

1,595

648

Board \& Executive

$\underline{199,478}$

$\underline{87,898}$

Programs - Schedule 3

300,615

189,873

INCREASE IN FUND BALANCE

INCOME - BOOKSHOP

FUND BALANCE, BEGINNING OF YEAR

$\underline{44,441}$

$\underline{43,036}$

FUND BALANCE, END OF YEAR

$\$ 46,323$

$\$ 44,441$

* Detailed financial statements are available from the SNHS office in Regina. 
ASSETS

1991

1990

CURRENT ASSETS

Cash

$\$ 81,934$

1,411

Cash - Bookshop

29,157

$\$ 46,056$

Accounts Receivable

Accounts Receivable - Bookshop

408

Investments

83,016

23,013

Inventory - Bookshop

$\underline{11,213}$

207,139

1,926

83,016

$\frac{9,600}{167,314}$

TRUST ACCOUNT INVESTMENTS (Note 2)

253,393

219,102

FLXED ASSETS

22,874

$\underline{19,995}$

$\$ 483,406$

$\$ 406,411$

LIABILITIES AND MEMBERS EQUITY

CURRENT LIABILITIES

Accounts Payable

Accounts Payable - Bookshop

$\$ 16,218$

$\$ 19,192$

786

1,517

Deferred Income (Note 4)

$\underline{40,215}$

22,065

57,219

42,774

LONG-TERM LIABILITIES

Specified Programs (Schedule 1)

103,597

80,099

Trust Accounts (Note 2)

253,393

$\underline{219,102}$

356,990

299,201

MEMBERS EQUITY

Fund Balance

46,323

44,441

Equity in Fixed Assets

$\frac{22,874}{69,197}$

$\frac{19,995}{64,436}$

$\$ 483,406$

$\$ 406,411$ 
A

Ablabesmyia illinoense 180

Ablabesmyia monilis 180

Ablabesmyia pulchripennis 180

Achillea megacephala - Large-headed Wooly

Yarrow 163

Acopa perpallida - Sharp-toothed Groundling

122

Acricotopus senex 181

Acronicta americana - American Dagger-

Moth 63

Acronicta dactylina - Fingered Dagger-Moth 63

Acronicta distans - Distans Dagger-Moth 64 Acronicta fragilis - Chain-dotted Dagger-

Moth 63

Acronictafuneralis - Funerary Dagger-Moth 64

Acronicta furcifera - Forked Dagger-Moth 63 Acronicta grisea - Gray Dagger-Moth 63

Acronicta haesitata 63

Acronicta hesperida - Large Gray Dagger-

Moth 63

Acronicta impleta - Powdered Dagger-Moth

63

Acronicta impressa - Impressa Dagger-Moth 64

Acronicta inclara - Unclear Dagger-Moth 63

Acronicta innotata - Unmarked Dagger-Moth

63

Acronicta interrupta - Interrupted Dagger-

Moth 63

Acronicta lanceolaria - Pointed Dagger-

Moth 64

Acronicta lepusculina - Cottonwood Dagger-

Moth 63

Acronicta lobeliae - Lobelia Dagger-Moth 63

Acronicta longa - Long-winged Dagger-

Moth 64

Acronicta mansueta - Gentle Dagger-Moth 63 Acronicta morula - Ochre Dagger-Moth 63

Acronicta noctivaga - Night-wandering

Dagger-Moth 64

Acronicta oblinita - Smeared Dagger-Moth 64

Acronicta quadrata - Quadrate Dagger-Moth

63

Acronicta radcliffei - Radcliffe's Dagger-

Moth 63

Acronicta sperata speratina - Sperata Dagger-

Moth 64
Acronicta spinigera - Nondescript Dagger-

\section{Moth 63}

Acronicta vulpina - Vulpina Dagger-Moth 63 Adam, Chris

Snow Geese over Buck Lake, photo 144

Waskesiu River, photo 114

Afotella cylindrica - Cylindrical Groundling

122

Agriopodes geminata - Gem Marvel 64 Agriopodes teratophora - Gray Marvel 64 Agroperina dubitans - Ruby Quaker 119 Agroperina helva - Yellow Three-spot 119 Agroperina inficita - Inficita Quaker 119 Agroperina lateritia - Red-winged Quaker 119

Agroperina lutosa - Muddy Quaker 119

Agroperina popofensis indela - Lined Quaker 119

Alberta, birds 93, lepidoptera, 183

Airdrie, birds 36

Beaverhill Lake, birds 79

Bertha Lake, lepidoptera 185

Big Lake (near St. Albert), birds 36

Bow River, birds 70

Bragg Creek, lepidoptera 186

Buck Mountain, lepidoptéra 186

Busby, birds 129

Calgary, birds $71,78,129$, lepidoptera 188

Canmore, birds 129

Claresholm, birds 129

Cooking Lake, birds 79

Cowley, birds 36

Del Bonita, lepidoptera 185

Dorothy, birds 93

Drumheller, lepidoptera 186

Edmonton, birds 129, lepidoptera 67

Elk Island National Park, birds 73

Fairview, birds 33

Goat Lake, lepidoptera 185

Grand Prairie, birds 73

Hanna, birds 93

Lamont, lepidoptera 122

Lethbridge, lepidoptera 122, 190

Manning, birds 73

Manyberries, lepidoptera 122

Medicine Hat, lepidoptera 122

Milk River, lepidoptera 185

Namaka Lake, birds 79

Peace River, lepidoptera 188

Red Deer, lepidoptera 188 
Richdale, birds 93

Seebe, Kananaskis, birds 129

Sundre, birds 122

Sunnynook, birds 93

Topaz Lake, birds 129

Turner Valley, birds 70

Vermilion Lakes, birds 129

Waterton Lakes National Park, birds 129 , lepidoptera 185

Writing-on-Stone Park, lepidoptera 122

Albinism, birds 31, 32, 139, 141

Alypia langtoni - Langton's Forester 65

Alypia octomaculata - Eight-spotted Forester 65

Amphipoea americana - American Ear Moth 121

Amphipoea interoceanica - Interoceanic Ear Moth 121

Amphipyra glabella - Gray Amphipyra 121

Amphipyra pyramidoides - Copper Under-

wing 121

Androloma maccullochii - Northern Forester 65

Andropolia contacta - Canadian Giant 121

Angelica arguta - Lyall's Angelica 188

Angelica dawsoni - Mountain Parsnip 188

Angelica genuflexa - Kneeling Angelica 188

Apamea alia - Dewy Quaker 119

Apamea amputatrix - Northem Quaker 119

Apamea apamiformis - Wild Rice Quaker 122

Apamea auranticolor - Brown-banded

Quaker 118

Apamea cinefacta - Gray Quaker 119

Apamea finitima - Pale-banded Quaker 119

Apamea impulsa - Southern Quaker 119

Apamea indocilis - Broken-banded Quaker

119

Apamea indocilis runata - Broken-banded

Quaker 119

Apamea inordinata - Montana Quaker 119

Apamea lignicolora - Wood-coloured Quaker

118

Apamea occidens - Western Quaker 122

Apamea parcata - Parcata Quaker 119

Apamea plutonia - Dusky Quaker 119

Apamea spadlingi - Spalding's Quaker 119

Af zmea verbascoides - Mullein Quaker 118

¿ipamea vultuosa - Airy Quaker 118

Apium graveolens - Garden Celery 188

Archanara oblonga - Oblong Sedge Borer

Moth 120

Archanara subflava - Suflava Sedge Borer

120

Armeria maritima ssp. interior - Inland Sea

Thrift 163
Artemisia dracunculus - Linear Leaved

Wormwood 187

Asai, J.

Monarchs, photo 149

Aseptis charata - Charata Quaker 121

Ash, Mountain 158

Atkinson, Edgar A., bird bander 59

Award, Cliff Shaw 224

Award, Conservation 224

1991 presentation, photo 225

Award, Larry Morgotch 224

1991 presentation, photo 225

B

Badger 44

Balsa labecula - White-blotched Balsa 121

Bancroft, Jean

1991 Cliff Shaw Award 224

Observations of albinism in birds. 139

Bancroft, Jean and Robert Parsons

Range expansion of the House Finch into the

Prairie Provinces. 128

Barton, Myron

Saskatchewan's North. 112

Bat 105

Bat, Big Brown 43

Bat, Little Brown 43

Baynton, Edgar, bird bander 59

Beaver 44

Belcher, Margaret

Elizabeth Roley Cruickshank 1895-1989 55

Bellura gortynoides - Brown-tailed Diver 121

Bellura obliqua - Pale-shouldered Brindle 121

Berger, Robert

Jean Bancroft, photo 225

Bird banding 3, 59

Bird count

Christmas, 49th Annual 7

Christmas update 100

Bittern, American 60

Bjorkland, Carol

Black Rosy Finch on Big Muddy CBC. 137

Blackbird, Brewer's 25, 60

Blackbird, Red-winged 25, 81

Blackbird, Rusty 25, 201, 214

Blackbird, Yellow-headed 139

Bluebird 158

Blue Jay 99

Bobcat 43

Book review

A prairie coulee. WILLOCK 108

Bird flight. BURTON 156

Birder's guide to southwestem Manitoba. CUTHBERT 217

Butterfly gardening - creating summer magic in your garden. XERCES SOCIETY/ 
SMITHSONIAN INST. 109

Extinct animals of the world. ROOTS 107

Field checklist of Saskatchewan birds (7th edition). KREBA 111

From the land of shadows - the making of Gray Ow1. SMITH 152
Chernovkskiia amphitrite 180

Chickadee, Black-capped 17

Chickadee, Boreal 16, 69

Chironomus anthracinus 180

Chironomus atrella 180

Chironomus atroviridis 180

Grasslands: the private hearings. POIRIER 53 Chironomus decorus 180

Hawks, eagles and falcons of North America. Chironomus plumosus 174 JOHNSGARD 154

Chironomus riparius 180

The great butterfly hunt - the mystery of the migrating monarchs. HERBERMAN 149

The nature fakers: wildlife, science and sentiment. LUTTS 150

Solitudes episode three: seasons in beaver country. (video) GIBSON 154

Vanishing animals of the world. ROOTS 107

Venomous animals of the world. ROOTS 107

Where the birds are. JONES 51

With ptarmigan and Tundra Wolves. HAMPSON 218

Braddell, David L

White-tail tail-wagging. 104

Brazier, Frank H., photo 41

Black-tailed Godwit sighted near Regina -

first for interior North America. 90

Chironomus staegeri 180

Chironomus tentans 180

Chytonix palliatricula - Cloaked Marvel 121

Cirsium arvense - Canada Thistle 71

Cladina mitis - Reindeer Lichen, photo cover No. 4

Cladonia coccifera - Red Apothelia, photo cover No. 4

Cladopelma viridulus 180

Cliff Shaw Award

Bancroft, Jean - 1991. 224

Collister, Douglas

Northern Mockingbird in Calgary, photo 71

Conchapelopia telema 180

Conservation, 220

Coot, American 60

Cormorant, Double-crested 27, 81

Cornus stolonifera - Red-osier Dogwood 71

Rosy Finch in Regina - Marjie's last bird. 40 Corynoneura celeripes 181

Mountain Plover sighting near Regina. 88

Brazier, Marjie, photo 41

memorial 40

Brindle, Pale-shouldered, photo 119

British Columbia, birds 87, 128, 131, lepidoptera 64, 189

Penticton, birds 128

Revelstoke, lepidoptera 67

Toobally Lakes, birds 73

Vancouver Island, mammals 215

Bryophaenocladius furcatus 174

Bufflehead 25

Bunting, Snow 16, 60, 100

Buzzard, Honey 211

Byersbergen, G.W.

C

Ring-billed Gulls, photo 85

Cardinal, Northern 133

Carlson, Muriel

Notes on Raccoons from Edam,

Saskatchewan. 101

Cat, Feral 43

Catbird, Gray 38

Catocala aholibah - Westem Underwing 66

Chase, Grant, photo 194

Chat, Yellow-breasted 38

Corynoneura sp. nr. taris 181

Cosmia calami - American Dun-bar 122

Cottontail, Eastern 44

Cottontail, Nuttall's 44

Coutu, Ken, photo 163

Lake Huron Tansy, photo 169

Mushrooms, photo 169

Coyote 44

Crambodes talidiformis - Verbena Moth 122

Crane, Sandhill 66, 81, 139

Crane, Whooping 66, 72

Crawford, Michael

Trumpeter Swan at Mystrey Lake, south of

Greenwater Lake Provincial Park, photo

cover No. 2

Creeper, Brown 16

Cricotopus bicintus 181

Cricotopus curtuss 181

Cricotopus intersectus 181

Cricotopus ornatus 174

Cricotopus politus 181

Cricotopus slossonae 181

Cricotopus sylvestris 181

Cricotopus sp. nr. tremulus 181

Cricotopus triannulatus 181

Cricotopus trifascia 181 
Crocodile, fossil 171

Crossbill, Red 25

Crossbill, White-winged 16

Crow, Amercian 5, 25, 60, 81, 123, photo 124

Cruickshank, Elizabeth Roley, photo 56

Elizabeth Roley Cruickshank 1895-1989. 55 Memorial 55

Crymodes burgessi - Burgess's Quaker 122

Crymodes devastator - Dest ructive Quaker 119

Crymodes longula - Mountain Quaker 119

Cryptochironomus conus 180

Cryptochironomus curryi 180

Cryptochironomus digitatus 180

Cryptochironomus eminentia 180

Cryptochironomus fulvus 180

Cryptochironomus ramus 180

Cryptochironomus scimitarus 180

Cryptochironomus stylifera 180

Cryptotendipes casuaris 180

Cryptotendipes darbyi 180

Cyphomella gibbera 180

D

Dagger-Moth, American, photo 62

Dagger-Moth, Ochre, photo 64

Dagger-Moth, Unmarked, photo 63

Dale, Bill

Blue Jays and window kills, letter 158

Deer, Mule 44

Deer, White-tailed 44, 104, 146, photo 145

Derotanypus alaskensis 180

Deschampsia mackenzieana - Mackenzie

Hair-grass 163

Diamesa cineralla 180

Dickson, Ross D.

Northem Mockingbird and Swamp Swallow overwinter in Calgary. 70

Dicrotendipes nervosus 180

Diptera

An amateur naturalist's guide to non-biting midges in Saskatchewan. 174

Chironomidae - non-biting midges 174

Dodge, Carman

Birds out my window. 81

Dove, Rock 16, 81, 100

Duck, American Black 25

Duck, Harlequin 25

Dutch Elm Disease, plants 117

Dypterygia rozmani - American Bird's-wing Moth 122

E

Eagle, Bald 16, 81, 100

Eagle, Golden 16, 81, 100

Egret, Cattle 36

Egret, Great 36
Egret, Snowy 37

Eichhorst, Bruce

Close-up of intermediate male, photo 198

Female Clark's Grebe mated with

intermediate male, photo 198

Eichhorst, Bruce A. and Barry D. Parkin

Clark's Grebes and suspected Westem x

Clark's Grebe hybrids in Manitoba. 196

Eider, Common 34

Eider, King 34

Einfeldia pagana 180

Elaphriafestivoides - Festive Midget 121

Elk, American 215

Elk 44, photo 216

Elm 117, photo 117

Worker finishes taking down an elm tree

infected with Dutch Elm Disease, photo 117

Enargia decolor - Angle-striped Sallow 121

Enargia infumata - Lesser Angle-striped

Sallow 121

Enargia mephisto - Mephisto Angle-striped

Sallow 121

Endochironomus nigricans 180

Eremobina claudens - Alberta Quaker 120

Eremobina jocasla - Jocasta Quaker 122

Ermine 43

Escaria homogena - Homogena Groundling 122

Eudocimus albus - White Ibis 142

Eudryas unio - Pearly Wood Nymph 65

Euplexia benesimilis - American Small

Angle Shades 121

Everit, Roy

Kestrel chicks, photo 75

Male Kestrel (female), photo 75

Male Kestrel shares incubating duties. 75

F

Falcon, Peregrine 25, 126

Falcon, Prairie 16

Felt, Harry L., bird bander 3

Financial statement

31 August 1991228

Finch, Black Rosy 25, 137

Finch, Brown-capped Rosy 40

Finch, Gray-crowned Rosy 25

Finch, House 128

Finch, Purple 25

Finch, Rosy 25, 40, 137

Findlay, C. Cliff

White Ibis sighting at Shoal Lake, Manitcba. 142

Fisher 44

Flicker, Common, 139

Flicker, Northem 16

Flycatcher, Acadian 206 
Flycatcher, Crested 61

Forester, Langton's, photo 65

Four Winds

White-tailed Deer near Chelan, Sask., photo 145

Fox, Red 44

Friesen, Victor

Crows and their (c)awing. 123

Frog, Northern Leopard, photo cover No. 3

G

Gadwall 4

Gannet, Northern 203

Gehlert, R. E.

It's coming..., photo 159

Glypotendipes barbipes 180

Glypotendipes lobiferus 180

Glypotendipes paripes 180

Gnatcatcher, Blue-grey 202

Godwit, Bar-tailed 91

Godwit, Black-tailed 90

Godwit, Hudsonian 91

Godwit, Marbled 59, 87

Gold, John A., bird bander 59

Goldeneye, Common 16, 81

Gollop, Bernie

Snow curves, photo 54

Goose, Barnacle 127

Goose, Brent 127

Goose, Canada 16, photo 157

Goose, Egyptian 206

Goose, Greylag 127

Goose, Snow 81, 126, 204, photo 144

Goose, Swan 206

Goose, White-fronted 81

Gopher, Northem Pocket 44

Goshawk, Northem 16, 100, 209

Grackle, Common 25, 98

Grebe, Clark's 196, 202, photos 198

Grebe, Eared 60

Grebe, Horned 4

Grebe, Red-necked 34

Grebe, Western 25, 202

Grebe, Western x Clark's 196

Grosbeak, Evening 16, 81, 100

Grosbeak, Pine 16, 70, 214

Grouse, Ruffed 16, 30

Grouse, Sage 25

Grouse, Sharp-tailed 5, 16, 100

Grouse, Spruce 25

Guillemot, Black 33

Guillemot, Pigeon 33

Gull, Black-backed 127

Gull, Black-tailed 203

Gull, Bonaparte's 76, 214

Gull, Franklin's 78, 81, 205
Gull, Glaucous 25, 205

Gull, Herring 81

Gull, Little 76, sketch 77

Gull, Ring-billed 81, 85, photo 85

Gull, Sabine 76

Gyrfalcon 25

H

Hare, Snowshoe 43

Harnischia curtilamellata 181

Harris, Wayne C.

Bohemian Waxwings, photo 26

Field checklist of Saskatchewan birds. 111

Harris, Wayne C., compiler

49th annual Christmas bird count: 1990. 7

Saskatchewan Christmas Mammal count: 1990.43

Christmas bird count update - Val Marie. 100

Harrisimemna trisignata - Harris' Three-spot 64

Hawk, Cooper's 25

Hawk, Ferruginous 5, 59, photo 155

Hawk, Red-tailed 5, 25

Hawk, Rough-legged 25, 100

Hawk, Sharp-shinned 5

Hawk, Sparrow 211

Hawk, Swainson's 4, 96

Hayesomyia senata 180

Helotropha reniformis - Kidney-spotted Rustic 120

Heracleum lanatum - Cow Parsnip 188, photo 186

Heron, Great Blue 81

Hicke, John

Grayson annual report. 158

Hjertaas, Dale

Unusual Barn Swallow nest site. 193

Holland, George.

Second albino Great Gray Owl sighted in

Manitoba. 32

Hooper, Donald F.

Barn Swallow nest on tractor cab, photo 195

Conservation Award, photo 225

First documented nesting for the Nashville Warbler in Saskatchewan. 68

Grant Chase puzzled by his haying companions, photo 194

Larry Morgotch Award, photo 225

Nesting swallows on the go. 194

Sap for survival. 201

Trumpeter Swans at Greenwater Lake, photo 73

Trumpeter Swans in eastern Saskatchewan. 72

Yellow-rumped Warbler feeding on sap, 
photo 201

Hi oper, Ronald R.

American Dagger-Moth, photo 62

Check-list of the moths of Saskatchewan part 8.62

Check-list of Saskatchewan moths part 9.118

European Skipper (Saskatchewan and New Jersey). photo 67

European Skipper in Saskatchewan. 67

Langton's Forester, photo 65

New Underwing Moth for the Prairie

Provinces. 66

Ochre Dagger-Moth, photo 64

Unmarked Dagger-Moth, photo 63

Houston, C. Stuart.

Saskatchewan bird banders: Harry L. Felt of Findlater, bander 007. 3

Saskatchewan bird banders: Baynton, Atkinson and Gold. 59

Hydraecia pallescens - Pallid Rustic 121

Hydraecia perobliqua - Oblique Rustic 121

Hypocoena basistriga - Base-streaked

Wainscot 120

Hypocoena defecta - Narrow-winged Borer 120

Hypocoena inquinata - Sordid Wainscot 120

Hypocoena rufostrigata - Brown-streaked

Rustic 120

Hyppa - Hyppa 121

I

Ibis, Glossy 202

Ibis, White 142

Ibis, White-faced 202

Ipimorpha pleonectusa - Even-lined Sallow 121

Ipimorpha subvexa - Subvexa Sallow 122

$\mathbf{J}$

Jackrabbit, Black-tailed 96

Jackrabbit, White-tailed 44

Jay, Blue $16,98,158$

Jay, Gray 16

Jay, Scrub 204

Jensen, Olaf Christian

Northern Leopard Frog, photo cover No. 3

Junco, Dark-eyed 16

James, Paul C.

A comment on incubating male falcons. 143

James, Paul C. and Keith Roney

New Double-Crested Cormorant colonies in

Saskatchewan. 27

James, Paul C. and Bob Kreba

Commentary: bird records in Saskatchewan and the Blue Jay. 99

Jonker, Peter

On site education: a first step to improved protection of endangered spaces. 220

Professor Stan Rowe explains landscape ecology of the S. Saskatchewan River Valley near Saskatoon, photo 221

Professor Stan Rowe on wind-exposed root of white spruce near William River, photo 223

K

Kestrel, American. 25, 30, 75, 139, 143, photo 75

Killdeer, 25, 60, 88

Kingbird, Eastern 4, 83

Kingbird, Western 61

Kinglet, Golden-crowned 25, 69

Kittiwake, Black-legged 202

Koes, Rudolph F.

Additions to the Manitoba Bird List (19851990). 202

Krivda, Walter

Murnmified bats at The Pas. 105.

L

Lahman, Fred W.

Canada Geese, photo 157

Crow, photo 124

Lamont, Sheila

Tribute 58

Landry, A.

Kettle Lake, Prince Albert National Park, photo 145

Lang, Anthony

Female Great Gray Owl on nest at Churchill, Manitoba, photo 208

Great Gray Owlets in the Churchill nest, photo 210

Habitat at the 1990 Churchill Great Gray Owl nest, photo 209

Northern Hawk Owl nest located in a brokenoff spruce, photo 212

Lang, Anthony L., James R. Duncan, Scott

Ramsay, and James D. Rising

Great Gray Owl and Northern Hawk Owl nests at Churchill, Manitoba. 208

Lark, Horned 16, 100

Lechea intermedia var. depauperata -

Impoverished Pineweed - 163

Lehman, Fred

Lesser Scaup, photo 86

Lepidoptera

Acroniclinae - Dagger-Moths 62

Agaristinae - Foresters 62

Alberta swallowtails and parnassians: natural history, keys and distribution. 183

Amphipyrinae - Quakers and Borers 118

Check-list of the moths of Saskatchewan part 8.62 
Check-list of Saskatchewan moths part 9.118

European Skipper in Saskatchewan. 67

New Underwing Moth for the Prairie

Provinces. 66

Lomatium dissectum - Mountain Wild

Parsnip 188

Lomatium triternatum - Western Wild

Parsnip 188

Longspur, Lapland 8

Loon, Common 81

Loon, Yellow-bellied 202

Luperina passer - Dock Rustic 119

Luperina stipata - Stipata Rustic 119

Lynch, Wayne

Deer Mouse, photo 144

Elk, photo 216

Ferruginous Hawk, photo 155

Northern Hawk Owl, photo 15

Untitled, photo 113

M

Winter scene, photo 138

Magpie, Black-billed 5, 16, 100, photo 162

Maine, birds 128

Mallard 4, 16, 60, 81

Mammal count

Saskatchewan Christmas 1990. 43

Manitoba, birds 76, 196, 202, lepidoptera 64

Altona, birds 130

Aweme, lepidoptera 122

Birtle, mammals 146

Blackfox Lake, birds 197

Carberry, birds 130

Cartwright, lepidoptera 122

Churchill, birds $78,202,208$, photos 208-210

Dauphin, birds 38

Delta, birds 139, 202

Delta Research Station, birds 73, 196

East Delta Marsh, birds 196

Grand Rapids, birds 78

Hudson Bay, birds 203

Lac du Bonnet, birds 29

Lake Dauphin, birds 78

Lake of the Woods, birds 79, 205

Lake Winnipegosis, birds 204

Leaf Rapids, birds 73

Lynn Lake, birds 73

Marshy Point, birds 199

Natalie Lake, birds 202

Norway House, birds 31,72 , photos 31

Oak Hammock Marsh WMA, birds 203

Oakbank, birds 130

Patricia Beach, birds 32, 76

Pelican Lake, birds 196, 202

Pinawa, birds 76

Poplar Point, birds 204
Riding Mountain National Park, birds 38, 202,215

St. Francois Xavier, birds 206

Shoal Lake, birds 142

Stonewall, birds 130

The Pas, mammals 105

Transcona, lepidoptera 65

University of Manitoba (campus), birds 126

Wabowden, birds 210

White Goose Lake, birds 211

Whitewater Lake, mammals 215

Winnipeg, birds $32,126,130,139,204$, lepidoptera 67

Map

location of 1990 counts, CBC 13

location of four areas on the East Delta Marsh, Manitoba, where Clark's

Grebes and intermediates were observed 196

Papilio canadensis 192

Papilio eurymedon 192

Papilio machaon 191

Papilio multicaudatus 192

Papilio zelicaon 191

Papilio zelicaon $x$ machaon 191

Parnassius phoebus 191

Reported sightings of the House Finch in the Prairie Provinces 132

Martin, Pine 43

Martin, Purple 139

Mason, P.G., P.W. Parker and P. Morrill

An amateur naturalist's guide to non-biting midges in Saskatchewan. 174

McCormick, Kevin J. and Jacques Sirois

Female scaup incubates Ring-billed Gull eggs. 85

Melit, Lawrence

Western Underwing, photo 66

Melit, Lorie

Copper Underwing, photo 120

Destructive Quaker, photo 118

Pale-shouldered Brindle, photo 119

Memorial

Elizabeth Roley Cruickshank 1895-1989. 55

Rosy Finch in Regina - Marjie's last bird. 40 Merganser, Common 25, 81

Merganser, Red-breasted 25

Merlin 16, 81

Merolonche lupini - Lupine Dagger-Moth 64 Messer, Deni-Vermai

A Crude Sound. 106

Massacre. 106

Mother Earth. 148

The Price of Progress. 106

Micropsectra dives 181 
Micropsectra nigripila 181

Micropsectra polits 181

Microtendipes caducus 181

Microtendipes pedellus 181

Mink 44

Mockingbird, Northern 38, 70, photo 71

Monarchs, photo 149

Moose 44

Moth, Leaf Roller 82

Mouse, Deer 44, photo 144

Mouse, House 44

Muskrat 44

$\mathbf{N}$

Nanocladius anderseni 182

Nanocladius crassocornis 182

Nanocladius spiniplenus 182

Nelson, John F.

Spotted Redshank sighted near Regina. 87

Nero, Robert W.

Blue Jay scavenging window-kills. 98

Focal concentration: a possible cause of mortality in the Great Gray Owl. 28

Oak Leaf Haven. 147

Peregrine Falcon attacks Snow Goose. 126

White Great Gray Owl. 31

New Brunswick, birds 134, lepidoptera 67

Newfoundland, birds 87, lepidoptera 67

Nighthawk, Common 81

Nilothauma babiyi 181

Northwest Territories, birds 33, 36

Baker Lake, birds 33

Cameron River, birds 33

Fort Good Hope, birds 33

Fort Simpson, birds 37

Fort Smith, birds 36

Great Slave Lake, birds 33, 36, 85

Hardisty Island, birds 34

Hay River, birds 36

Nahanni, birds 73

Yellowknife, birds 33

Nuthatch, Red-breasted 16

Nuthatch, White-breasted 16

O

Odocoileus virginianus - White-tailed Deer 146

Oligia bridghami - Bringham's Brocade 120

Oligia egens - Neumogen's Quaker 120

Oligia fractilinea - Lined Stalk-borer 120

Oligia illocata - Wandering Quaker 120

Oligia mactata - Prairie Quaker 120

Oligia modica - Black-banded Brocade 120

Oligia oblusa - Obtuse Quaker 120

Oligia tonsa - Short-cloaked Quaker 120

Ontario, birds 87

Guelph, birds 133
Hudson Bay, birds 78

James Bay, birds 78, 203

London, lepidoptera 67

Moosonee, birds 203, 211

Niagara-on-the-Lake, birds 130

Oriole, Northern 82

Orthocladius carlatus 182

Orthocladius mallochi 182

Orthocladius nigritus 182

Orthocladius obrumbatus 182

Orthocladius rivicola 182

Orthocladius robacki 182

Osprey 81

Otter, River 43

Owl, Barred 25, 28

Owl, Burrowing 93

Owl, Great Horned 5, 16, 30, 69, 100

Owl, Great Gray 25, 28, 31, 32, 139, 208, photos cover No. 1, 31, 208-210

Owl, Northern-Hawk 25, 29, 208, photo 15 , (nest) 212

Owl, Nonhern Saw-whet 25

Owl, Short-eared 16, 100, 211

Owl, Snowy 16, 127

Owl, Ural 211

$\mathbf{P}$

Palaentology 171

Papaipema aweme - Aweme Borer Moth 122

Papaipema cataphracta - Burdock Borer Moth 121

Papaipema circumlucens - Hops Borer Moth 121

Papaipema furcata - Ash Tip Borer Moth 122

Papaipema harrisii - Cow Parsnip Borer Moth 121

Papaipema leucostigma - Columbine Bører 121

Papaipema marginidens - Water HEirilock Borer Moth 121

Papaipema nelita - Nelita Borer Moth 122

Papaipema rigida - Rigid Sunflower Borer Moth 122

Papaipema unimoda - Meadow Rue Borer Moth 121

Papaipema verona - Verona Borer Moth 122

Papilio bairdii brucei 187

Papilio canadensis 183

Papilio eurymedon 183

Papilio machaon 183

Papilio machaon dodi, photo 186

Papilio multicaudatus - Two-tailed

Swallowtail 190

Papilio rutulus - Westem Tiger Swallowtail 189

Papilio zelicaon 183, photos 186 
Parachironomus abortivus 181

Parachironomus frequens 181

Parachironomus tenuicaudatus 181

Paracladopemla nereis 181

Paracladopemla winnelli 181

Parakiefferiella tortulata 182

Paralauterborniella nigrohalterale 181

Parastichtis discivaria - Mottled Quaker 120

Paratanytarsus confusus 181

Paratanytarsus laccophilus 181

Paratendipes albimanus 181

Parnassian 183

Parnassius clodius 184

Parnassius phoebus 183

Parnassius phoebus smintheus 183

Partridge, Gray 6, 16, 100, photo 11

Partridge, Hungarian 6

Pastinaca sativa - Garden Parsnip 188

Pelican, White 81, photos 42, 156

Peronne, Marie

Black Swallowtail, letter 192

Petasites palmatus - Palmate-leaved

Coltsfoot 187

Phaenopsectra obediens 181

Pheasant, Ring-necked 16, 100

Phlogophora iris - Olive Angle Shades 121

Phlogophora periculosa - Great Angle

Shades 121

Phosphila miselioides - Spotted Phosphila 121

Photo

Members of group guided through the

Athabasca Sand Dunes, 1991, 222

Picea pugens - Colorado Spruce 133

Pintail 4

Pintail, Northern 59

Pipit, Sprague's 59

Plagimimcus expallidus - Expallidus Brown 122

Platyperigia extima - Civil Rustic 121

Platyperigia meralis - Mooned Rustic 121

Platysenta discinstringa - Streaked

Groundling 122

Platysenta videns - White-dotted Groundling 122

Plover, Mountain $₹ 8$

Poem

A Crude Sound 106

Massacre 106

Mother Earth 148

Oak Leaf Haven 147

The Price of Progress 106

Saskatchewan's North 112

Polypedilum sp. nr. aviceps 181

Polypedilum convictum 181
Polypedilum digitifer 181

Polypedilum fallax 181

Polypedilum illinoense 181

Polypedilum laetum 181

Polypedilum obtusum 181

Polypedilum scalaenum 181

Populus balsamifera - Balsam Poplar 71

Porcupine 44

Potthastia longimana 180

Procladius bellus 180

Procladius denticulatus 180

Procladius freemani 180

Procladius nietus 180

Pronghom 44

Protagrotis extensa - Dark white-veined Quaker 119

Protagrotis niveivenosa - White-veined Quaker 119

Protoperigia posticata - White-winged Quaker 122

Proxenus miranda - Glistening Rustic 121

Psectrocladius flavus 182

Psectrocladius simulans 182

Psectrotanypus alaskensis 180

Psectrotanypus dyari 180

Pseudanarta crocea - Yellow Orange-wing 121

Pulson, J.

White Pelicans, photo 156

Q

Quaker, Dest ructive, photo 118

Quebec, birds 134, lepidoptera 67

R

Rabbit, Black-tailed Jack 96

Raccoon 44, 101, photo 102

Radwanski, Elaine

White-headed Redpoll. 141

Redpoll with hood, photo 141

Rail, Black 203

Raven, Common 16, 124, 209

Raven, Northern 81

Redhead 4

Redpoll, Common 16,70, 141, photo 141

Redpoll, Hoary 16, 141

Redshank, Spotted 87

Redstart 68

Rheotanytarsus exiguus 181

Rhizagrotis albalis actona - Actona Sallow 122

Roadrunner, Greater 204

Robackia claviger 181

Robackia demeijerei 181

Robin, American 16, 60, 81, 98

Ross, $\mathrm{S}$.

Fiery sunrise, Prince Albert National Park, 
photo 112

Rowe, Professor Stan, photos 221, 223

Roy, Frank, photo 163

Abundance of cones on a black spruce, photo 166

Aerial view of dunes east of the William River, photo 164

Dennis Tyson and George Tosh look to the William River Dunes, south shore Lake Athabasca, photo 166

Desert sands and water, photo 165

Dunes rise above the William River, photo 167

Felt-leaved Willow, photo 169

"Gobi" desert pavement, photos 168

Horsehead landmark, Little Gull Lake, photo 170

Pine clings to life above the William River, photo 165

Sands move on, photo 166

Slow-moving sand overtakes muskeg and forest, Little Gull Lake, photo 164

$\mathbf{S}$

Saetheria tylus 181

Salamander, Tiger 94

Salix brachycarpa var psammophila - Short-

fruited Sand Willow 163

Salix silicicola - Felt-leaved Willow 163

Salix turnorii - Turnor's Willow 163

Salix tyrrelli - Tyrrell's Willow 163

Sandpiper, Buff-breasted 61

Sandpiper, Stilt 90

Sandpiper, Upland 59

Sapsucker, Yellow-bellied 68, 201

Saskatchewan, birds 99, diptera 174, lepidoptera 79, 118, plants 117

Abernethy-Katepwa Lake CBC 8, CMC 44

Anglin Lake, lepidoptera 120

Archerwill, birds 141

Assiniboia CBC 8, CMC 44, lepidoptera 67 mammals 103

Athabasca Sand Dunes, 163, photos 164-170, 222

Aylsham, lepidoptera 64, 118

Bainbridge, lepidoptera, 121

Bangor, CBC 8, CMC 44

Battleford, CBC 26

Battlefords Provincial Park, lepidoptera 63, 121

Belbutte, birds 60

Bertwell, lepidoptera 64

Bethune, birds 5, CBC 8, CMC 44

Big Beaver, birds 137

Big Muddy Lake, birds 137, CBC 8, CMC 44, lepidoptera 63
Big Muddy Valley, CBC 8, CMC 44

Big Piwei Lake, birds 69

Big River, CBC 8, CMC 44

Biggar, CBC 8, CMC 44

Birch Hills, CBC 8, CMC 44

Bird bander 3, 59

Bjork Lake, birds 73

Bjorkdale, lepidoptera 121

Blaine Lake, mammals 103

Broadview, $\mathrm{CBC} 8$

Bromhead, CBC 8, CMC 44

Buffalo Pound Lake, birds 5

Buffalo Pound Provincial Park, lepidoptera 63,121

Candle Lake, CBC 8, CMC 45, mammals 103

Carlyle, mammals 102

Carragana, birds 69, CBC 8, CMC 45

Carrot River, CBC 26, palaentology 171, plants 117

Chelan, photo 145

Christopher Lake, mammals 103

Churchill Lake, birds 27

Clearwater River, lepidoptera 65, 121

Crane Lake, birds 59

Creighton, lepidoptera 119

Crooked Lake, CBC 8, CMC 45

Cumberland House, lepidoptera 63, 119

Cutknife, lepidoptera 122

Cypress Hills, birds 73, diptera 177 , lepidoptera 63,185

Deschambault Lake, birds 78, lepidoptera 119

Devil's Lake, CBC 8

Dilke, birds 6, CBC 8

Disley, mammals 102

Douglas Provincial Park CBC 8, CMC 45, lepidoptera 64

Duck Mountain Provincial Park, lepidoptera 120

Dundurn (Blackstrap Lake), birds 78

Duval, CBC 8, 25, CMC 45

Earl Gray, lepidoptera 63, 121

Eastend, CBC 9, CMC 45

Echo Lake, birds 74

Edam, CBC 9, CMC 45, mammals 101

Endeavour, $\mathrm{CBC} 9$

Erwood, lepidoptera 63

Estevan, birds 193, CBC 9, CMC 45, mammals 102 , plants 117

Eston, lepidoptera 65

Estuary, lepidoptera 121

Fife Lake, CBC 9, CMC 45

Findlater, birds 3

Fond du Lac River, birds 73

Fort Esperance, lepidoptera 121

Fort Qu'Appelle, birds 131, CBC 9, CMC 
46 , lepidoptera $63,66,118$

Fort Walsh, CBC 9, CMC 46

Foster Lake, lepidoptera 63

Frenchman River Valley, CMC 43

Gardiner Dam, CBC 9, CMC 46

Geikie River, lepidoptera 121

Glen Ewen, lepidoptera 63, 121

Good Spirit Lake, CBC 9, CMC 46

Gordon Lake, lepidoptera 121

Govenlock, CBC 9, CMC 46

Grand Centre, CMC 46

Grand Centre-Pierceland, CBC 9

Grand Coulee, plants 117

Grasslands National Park, CBC 9, CMC 46, diptera 177

Grayson, letter 158

Great Sand Hills, birds 59

Greenbush Campsite, lepidoptera 65

Greenbush River, birds 68

Greenwater Provincial Park, birds 69, 72 , photo cover No. 2, 73

Halbrite, lepidoptera 118

Harlan, lepidoptera 120

Hudson Bay, birds 68, 73, CBC 9, CMC 46, lepidoptera 63,120

Humboldt, CBC 9, CMC 46

Ile-a-la-Crosse, mammals 102

Indian Head, CBC 9, CMC 47, mammals 103

Kamsack, CBC 9, CMC 47, lepidoptera 63

Kazan Lake, birds 69

Kelvington, CBC 9

Kenaston, CBC 9, CMC 47

Kettle Lake, photo 145

Killdeer, lepidoptera 119

Kindersley, CBC 9, CMC 47

Kinistino, birds 6

Kutawagan Lake, CBC 9, CMC 47

La Loche, lepidoptera 65

La Ronge, CBC 9, CMC 47, lepidoptera 63, 119

Lac des Isles, birds 73

Lake Athabasca, birds 78, diptera 177, photo 166

Lake Diefenbaker, CBC 7

Last Mountain Lake, birds 5, 199

Last Mountain Lake WMU, CBC 9, CMC 47

Leader, CBC 9, CMC 47

Limerick, lepidoptera 121

Little Gull Lake, birds 78, photos 164, 170

Little Nut Lake, birds 131

Livelong, CBC 9

Loon Lake, lepidoptera 120

Love, lepidoptera 63, 119

Love-Torch River, CBC 9, CMC 47

Lumsden, birds 4, lepidoptera 64, 122
Luseland, CBC 9, CMC 48

Maidstone Bridge, CBC 9, CMC 48

Maple Creek, birds 59

Marean Lake, birds 72

Marsden, CBC 9, CMC 48

Maryfield, lepidoptera 63, 121

Meadow Lake, mammals 102

Meadow Lake Provincial Park, lepidoptera 63,120

Medstead, birds 60

Melfort, lepidoptera 120

Melville, CBC 9, CMC 48

Milliken Lake, lepidoptera 65

Mirond Lake, birds 73

Missinipe, CBC 9

Montreal Lake, birds 73

Montreal River, birds 69

Moose Jaw, CBC 9, CMC 48, lepidoptera 63

Moose Mountain, lepidoptera 63, mammals 102

Moose Mountain Provincial Park, lepidoptera 63

Mullinger, mammals 103

Mystrey Lake, birds 74, photo cover No. 2

Neely Lake, CBC 10

Nipawin, lepidoptera 63

Nipawin Provincial Park, lepidoptera 63, 119

Norquay, lepidoptera 120

North Battleford, birds 73, mammals 101

North Saskatchewan River, birds 81, mammals 101

Northside, mammals 103

Old Wives Lake, lepidoptera 119

Onion Lake, lepidoptera 121

Otter Rapids, lepidoptera 63

Outlook, CBC 7

Outlook Regional Park, lepidoptera 121

Oxbow, lepidoptera 121, 122

Pelican Narrows, birds 73

Peter Pond Lake, birds 27

Piapot, birds 60

Pike Lake, CBC 10, CMC 48, lepidoptera 63

Pine Island, mammals 101

Piwei River, birds 194

Porcupine Plain, birds 72, CBC 10

Porter Lake, lepidoptera 63

Prince Albert, birds 81, CBC 10, CMC 48, lepidoptera 64,120

Prince Albert National Park, birds 69, CBC 10, CMC 48, lepidoptera 64 , photos $112-114,145$

Punnichy, lepidoptera 119

Qu'Appelle Dam, CBC 10, CMC 48

Qu'Appelle Valley, birds 74, mammals 102

Rabbit Lake, birds 60 
Radville, birds 60

Ravenscrag, lepidoptera 121

Raymore, CBC 10, CMC 49

Red Earth, lepidoptera 120

Regina, birds $33,40,78,83,87,88,90,131$, 193, CBC 10, CMC 49, lepidoptera 65 ,

119, mammals 102, palaeontology 172

Richardson, birds 88

Rocanville, plants 117

Roche Percee, lepidoptera 63, 119

Rosefield, lepidoptera 63, 122

Rosthem, mammals 103

Round Lake, CBC 10, CMC 49, lepidoptera 122, mammals 103

Saskatchewan Landing Provincial Park, lepidoptera 119

Saskatchewan River, diptera 174

Saskatoon, birds 95, 96, 131, CBC 10, CMC 49, diptera 177, lepidoptera 119

Scott, CBC 10, CMC 49

Shady Lake, photo 114

Shaunavon, plants 117

Shoal Lake, lepidoptera 119

Skull Creek, CBC 10, CMC 49

Snowden, CBC 10, CMC 49

Somme, birds 131, 195, 201, CBC 10, lepidoptera 64, 121

South Saskatchewan River Valley (near

Saskatoon), photo 221

Spalding, CBC 11, CMC 49

Spinney Hill, CBC 11, CMC 49

Spring Valley, CBC 11, CMC 50

Squaw Rapids, birds 74, CBC 11, CMC 50, mammals 103

Stony Rapids, birds 73

Sturgeon Landing, lepidoptera 63, 121

Swift Current, CBC 11, CMC 50, lepidoptera 64,122

Tantallon, lepidoptera 63, 118

Tisdale, CBC 11

Tobin Lake, diptera 174

Torch River, birds 73

Trossachs, lepidoptera 63

Turte Lake, CBC 11

Turtlelake River, mammals 101

Uranium City, lepidoptera 65

Val Marie, birds 88, CBC 100, lepidoptera 121

Valeport, birds 74

Vawn, mammals 101

Veillardville, birds 73

Wadena, lepidoptera 120

Waldsea Lake, diptera 174

Wasekamio Lake, birds 27

Waskesiu River, diptera 174, photo 114
Weekes, lepidoptera 64, 121

Weybur, birds 131,193, CBC 11, CMC 50

White Bear, CBC 11, CMC 50

White Bear Lake, CBC 11, CMC 50

Whitebeech, CBC 11, CMC 50

Whitewood, CBC 11, CMC 50

William River, 163, photos $164,165,167$, 168,223

Woodlawn Regional Park, plants 117

Yorkton, CBC 11, CMC 50

Scaup, Greater 85

Scaup, Lesser 4, 25, 85 , photo 86

Schmidt, A.

Gray Partridge, photo 11

Schmutz, Josef K., Gwendolyn Wood

and Daniel Wood

Spring and summer prey of Burrowing Ow in Alberta. 93

Schroeder, Curt

Frank Switzer presents award to

Don Hooper, photo 225

Scott, Lorne

Black-billed Magpie, photo 162

Elizabeth Roley Cruickshank, photo 56

Land clearing, photo 148

Seib, Gary W.

Raccoon, photo 102

Shelduck, 127

Shoveler, Nonthern 4, 59

Shrew, Masked 44

Shrike, Loggerhead 60

Shrike, Northern 16, 100

Simyra henrici - Henry's Marsh Moth 64

Sirois, Jacques

First record of the Black Guillemot near

Yellowknife, Northwest Territories. 33

Sirois, Jacques, George Low, and John

Turquand McCollum

First Great Egret record in the

Northwest Territories. 36

Siskin, Pine 16

Sium suave - Water Parsnip 188

Skipper, European 67

Skunk, Striped 29, 44

Solitaire, Townsend's 25

Sparrow, American Tree 16

Sparrow, Brewer's 202

Sparrow, Eurasian Tree 206

Sparrow, Fox 25, 201

Sparrow, Harris' 25

Sparrow, House 16, 30, 81, 82, 98, 100, 134, $139,158,206$, photo 140

Sparrow, Song 25

Sparrow, Swamp 70

Sparrow, Vesper 206 
Sparrow, White-throated 25,68

Spartiniphaga includens - Silver-C Borer 120

Spartiniphaga inops - Single-spot Borer 120

Spartiniphaga panatela - Panatela Borer 120

Sperling, Felix

3rd instar of Papilio zelicaon on Heracleum lanatum, Buck Mountain, Alberta, photo 186

5th instar of Papilio zelicaon on Angelica leaf, photo 186

Papilio machaon dodi, Drumheller, Alberta, photo 186

Sperling, Felix and Norbert Kondla

Alberta swallowtails and parnassians: natural

history, keys, and distribution. 183

Spodoptera frugiperda - Fall Armyworm

Moth 122

SPRR

Worker finishes taking down an elm infected with Dutch Elm Disease, photo 117

Squirrel, Fox 44

Squirrel, Grey 44

Squirrel, Ground 96

Squirrel, Northern Flying 44

Squirrel, Red 44, 103

Squirrel, Richardson's Ground 44, 93

Starling, European 16, 81

Stellaria arenicola - Sand Chickweed - 163

Stenochironomus hilarus 181

Stibadium spumosum - Frothy Moth 122

Stinson, Cst. Jim

Great Gray Owl, near Norway House, MB,

photo cover No. 1, photo 31

Stiria rugifrons - Yellow Groundling 122

Strange, K.

Aspen stand, Prince Albert National Park, photo 113

Swallow, Bam 59, 81, 193, photo (nest) 195

Swallow, Cliff 4

Swallow, Tree 158

Swallowtail 183

Swallowtail, Black 192

Swan, Mute 203

Swan, Trumpeter 72, photo cover No. 2

Swan, Tundra 25, 72, 81, 203

Switzer, Frank

Copy-cat feeding of House Sparrows. 82

Jim Elliott presents Don Hooper with award, photo 225

Long-tailed Weasel, photo 43

Pat Adams accepts award for Most Birds on a Club List for Saskatoon NHS, from Dick Gutfriend of Weyburn NHS. Stan Shadick accepts award for Most Birds on an Individual's List on behalf of
Burke Korol, photo 226

Sand hills near Webb, photo 35

Tribute to Sheila Lamont, 58

White Pelican, photo 42

Synorthocladius semivirens 182

Syroteuk, Merv

Shady Lake, Prince Albert National Park, photo 114

$\mathbf{T}$

Tanacetum huronense var floccosum - Lake

Huron Tansy - 163

Tanager, Scarlet 38

Tansy, Lake Huron, photo 169

Tanypus punctipennis 180

Tanytarsus glabrescens 181.

Tanytarsus guerlus 181

Taraxacum officinanale - Dandelion 189

Taylor, Emest

Albino House Sparrow, photo 140

Taylor, Peter

Little Gull in southern Manitoba and adjacent regions, with notes on juvenile plumage. 76

Little Gull - juvenile plumage, sketch 77

Taylor, Robert R.

Great Gray Owl, near Lac du Bonnet, MB, photo 29

Teal, Blue-winged 4

Teal, Green-winged 4, 59

Tem, Black 81

Thienemanniella cf. xena 182

Thrasher, Brown 38, 70

Thrush, Hermit 201

Thrush, Swainson's 98

Thrush, Varied 25, 40

Thrush, Wood 40

Thymelicus lineola - European Skipper 67

Tokaryk, Tim T.

Cutbank of the Carrot River, photo 171

Fossil crocodile discovered along the Carrot River. 171

The skull, turned upside lown, in the rock, photo 172

Tosh, George, photos 163, 166

Red Apothelia, Cladonia coccifera (L.)

Willd., nestled in Reindeer Lichen, Cladina mit is (Sandst.) Hustich, photo cover No. 4

Turnor's Willow, photo 169

William Lake, photo 168

Tosh, Margaret

Members of party to the Athabasca Sand

Dunes, photo 163

Trachea delicala - Delicate Trachea 121

Trichoplexia exornata - Northem Banded

Quaker 119 
Turkey, Wild 25

Tvetenia vitracies 182

Tyson, Dennis, photos 163, 166

U

Underwing, Copper, photo 120

Underwing, Western, photo 66

Urited States

Alabama, birds 88

Alaska, birds 36

California, birds 88, 204, diptera 174

Colorado, lepidoptera 67

Connecticut, birds 87

Florida, birds 89, diptera 174

Hawaii, birds 139

Idaho, birds 73, 88, 128, 137, 204

Illinois, birds 128, lepidoptera 67

Iowa, birds 126

Kansas, birds 204

Louisiana, birds 4

Maine, birds 134

Maryland, birds 128, 204

Michigan, birds 88, lepidoptera 67

Minnesota, birds 4, 73, 79, 129, 213, lepidoptera 67

Mississippi, birds 88

Montana, birds 72, 79, 88, 128, 137 , lepidoptera 64,122

Nebraska, birds 4

Nevada, birds 73, 87, 88, 137

New Hampshire, birds 134

New Jersey, birds 139, lepidoptera 67

New York, birds 128, lepidoptera 67

North Dakota, birds 79, 204, lepidoptera 66, 67

Ohio, lepidoptera 67

Oregon, birds $73,88,128$, lepidoptera 64

Pennsylvania, birds 87

Rhode Island, birds 87

South Carolina, birds 128

South Dakota, birds 4, 73, 79, 128, lepidoptera 66

Texas, birds 5

Utah, birds 137, 199, lepidoptera 65

Vermont, birds 134

Virginia, birds 88

Washington, birds 89,128

Wisconsin, birds 128,139 , lepidoptera 67

Wyoming, birds $72,88,128,204$

V

Vermont, birds 134

Vireo, Red-eyed 38, 68

Vireo, Solitary 68

Vole, Gapper's Red-backed 44, 213

Vole, Meadow 44
Vole, Northern Red-backed 213

Vulture, Turkey $25,81,127$

W

Wagtail, Yellow 204

Walley, William J.

Elk calf "hides" underwater. 215

White-tailed buck attempts to mate with wounded doe. 146

Yellow-breasted Chat near Riding Mountain National Park, Manitoba. 38

Warbler, Blackburnian 68

Warbler, Black-throated Green 68

Warbler, Blue-winged 204

Warbler, Chestnut-sided 68

Warbler, Connecticut 68

Warbler, Magnolia 69

Warbler, Mourning 68

Warbler, Nashville 68

Warbler, Prairie 205

Warbler, Prothonotary 205

Warbler, Yellow 68

Warbler, Yellow-throated 205

Warbler, Yellow-rumped 68, photo 201

Waterthrush, Northern 68

Waxwing, Bohemian 16,70,158, photo 26

Waxwing, Cedar 16, 81, 82

Weasel, Least 44

Weasel, Long-tailed 44, photo 43

Weyburn Birdathon Awards 226

Wheatear, Northern 202

Widgeon, American 139

Willet 87

Willow, Felt-leaved, photo 169

Willow, Turnor's, photo 169

Wolf, Timber 44, 104

Woodpecker, Black-backed 25

Woodpecker, Downy 16, 158

Woodpecker, Hairy 16, 158

Woodpecker, Pileated 16, 38

Woodpecker, Three-toed 25

Wren, House 60, 158

Wren, Winter 68

Y

Yellowlegs, Greater 87, 90

Yellowlegs, Lesser 87

Yellowthroat, Common 68

Yukon, birds 73

$\mathrm{X}$

Xenochironomus scopulus 181

Xenochironomus xenolabis 181

Xylomoia chagoni - Chagnon's Quaker 120

$\mathrm{Z}$

Zizia aptera - Heart-leaved Alexanders 188 


\section{MEMBERSHIP RENEWAL AND GIFT FORM}

Name

Address

City/Town

Prov.

Postal Code

MOVING? To avoid missing any issues of the Blue Jay, please write your old address above, and your new address below:

Name

Address

City/Town

Prov

Postal Code

Effective

Membership Type (Please check) (Prices listed in Canadian funds)

Regular - Canada $(\$ 15)$

Regular - outside Canada $(\$ 18)$

No. of family members

Senior Citizen - Canada $(\$ 13)$....

An income tax receipt for Life,

Patron, or Sustaining Membership

and Donations - check here

I am a new mer iber for 199

This is a renewal for 199

I vish to make $a$ donation to:

Conservation Fund \$

Heritage Marshes Fund \$

Other $\$$

Check above if receipt is required.

* Life membership is payable at one time, or over five years, or any shorter period agreed.

* Any amount over $\$ 10$ in excess of the regular membership of $\$ 15$ is viewed as a donation for income tax purposes and a receipt for the excess amount will be mailed if the appropriate box is checked above.

* Bulk subscriptions to schools and clubs are $\$ 15$ for the first subscription and $\$ 13$ for each additional subscription to the same address (minimum of five subscriptions).

Please send Blue Jay Gift Subscription to:

Name

Address

City/Town

Prov

Postal Code

[] Check here if you wish us to send a card announcing the gift.

Typa of membership (Please check) (Prices listed in Canadian funds)

!n dividual - Canada $(\$ 15)$

Family - Canada $(\$ 20)$.

Youth - Canada $(\$ 8)$

[] Individual - Outside Canada (\$18)

No. of family members

Senior - Canada $(\$ 13)$

"Send renewal form to

\section{SNHS}

Box 4348

Regina, Saskatchewan

S4P 3W6

Mi te cheques and money orders payable to Saskatchewan Natural History Society.

Your donation will be acknowledged in Blue Jay News by name only. Check here if you do not wish to be acknowledged. []

* Do you know of any person interested in natural history and conservation who does not receive the Blue Jay? Please send their name and address and we will send a sample Blue Jay and an invitation to join our Society. 